\title{
Whole-genome sequencing combined with a case-control study of an outbreak of staphylococcal food- poisoning
}

Ae Kyung Park ${ }^{1}$, Jungsun Park ${ }^{1}$, Eunkyung Shin ${ }^{1}$, Soojin Kim ${ }^{1}$, Hyun Ju Jung ${ }^{1}$, Jeong-Hoon Chun ${ }^{1}$, Junhyuk Park $^{2}$, Hyunah Lee ${ }^{2}$, Yeonjeong Kim ${ }^{3}$, Cheonhyeon $\mathrm{Kim}^{3}$, Hyun Ja Oh${ }^{4}$, Kyu Jam Hwang ${ }^{1}$ and Junyoung Kim ${ }^{1 \S}$ ${ }^{1}$ Division of Bacterial Diseases, Center for Laboratory Control of Infectious Diseases, Korea Centers for Diseases Control and Prevention, Chungcheongbuk-do, Republic of Korea

${ }^{2}$ Division of Microbiology, Chungcheongnam-do government research institute of public health and environment, Chungcheongnam-do, Republic of Korea

${ }^{3}$ Division of Infectious Diseases Investigation, Jeollabuk-do government research institute of public health and environment, Jeollabuk-do, Republic of Korea

${ }^{4}$ Boryenong public health center, Chungcheongnam-do, Republic of Korea

${ }^{\S}$ Corresponding author: Junyoung Kim

Division of Bacterial Diseases, Center for Laboratory Control of Infectious Diseases, Korea Centers for Diseases Control and Prevention, Cheongju-si, Chungcheongbuk-do, 28159, Republic of Korea

Tel: +82-43-719-8116; Fax: +82-43-719-8109; E-mail: jun49@hanmail.net

Running Title: Staphylococcal food poisoning outbreak in South Korea

\begin{abstract}
This study reports an outbreak of Staphylococcus aureus food poisoning in Chungcheongnam-do, Korea, in 2018; a case-control study was conducted among participants of a village festival after they consumed a commercially catered buffet lunch. Whole-genome sequencing (WGS) was performed for in silico molecular typing and to investigate the genetic relationships among the $S$. aureus isolates. The analysis of the clustering of isolates identified by using WGS allowed us to identify a source of the outbreak. In silico multilocus sequence typing (MLST) and Staphylococcus aureus protein A (spa) typing analyses indicated that the majority of $S$. aureus isolates, including those from patients, food, and a food handler, showed identical sequence types (STs) and spa types (MLST6 and spa t304, respectively). The phylogenetic results showed their genetic relationship, suggesting that the contamination causing the outbreak most likely originated from the food handler. In this report, we combined WGS data with information from a case-control study to investigate a foodborne disease This peer-reviewed article has been accepted for publication but not yet copyedited or typeset, and so may be subject to change during the production process. The article is considered published and may be cited using its DOI 10.1017/S0950268820001132.
\end{abstract}

Epidemiology \& Infection is published by Cambridge University Press. 
outbreak caused by S. aureus in Korea. This approach is a major improvement in foodborne disease outbreak detection and defines pathogen sources throughout the food chain.

Keywords: Staphylococcus aureus, outbreak, foodborne, whole-genome sequencing (WGS), case-control study

\section{Introduction}

Foodborne diseases are major public health concerns worldwide [1]. Staphylococcal food poisoning (SFP) is one of the most common causes of foodborne illness in the world [2-4]. Each year in the United States, more than 240,000 people develop SFP [5], and in Europe, the number of SFP outbreaks reported by the European Food Safety Authority (EFSA) was 114 SFP outbreaks in 2018, 2.2\% of which were associated with reported bacterial toxins [6]. In Korea, from 2002 to 2011, 174 SFP outbreak cases were reported, accounting for $7.4 \%$ of all foodborne infection outbreaks [7]. In recent decades (2007-2016), on average, 13.1 foodborne illness outbreaks involving 344.6 patients per year have been documented [8].

Staphylococcus aureus produces a wide variety of toxins, including staphylococcal enterotoxins (SEs), which are causative agents of SFP [9]. To date, 23 SEs have been reported [10], and a wide variety of SEs causing SFP outbreaks in humans have been reported $[11,12]$. Food handlers who carry enterotoxigenic staphylococci in their nostrils or on their hands are regarded as the main source of food contamination [13]. The onset of SFP symptoms commonly occurs between 0.5 and 6 hours, and illness typically lasts for 1 day (up to 3 days), with rapid recovery [14]. However, staphylococcal infections can be life threatening when they become systemic and spread to vital body organs $[15,16]$.

For the effective and rapid control of outbreaks, the early detection of outbreaks is necessary, and molecular typing has played an important role in outbreak investigations. With the rapid advances in technology in recent years, new molecular techniques, such as next-generation sequencing (NGS), have been applied to investigate outbreaks [17-19]. The use of NGS in outbreak analysis facilitates rapid and accurate identification of the pathogen and identifies the transmission patterns of the pathogen, helping rapidly control an outbreak [20, 21]. In Korea, S. aureus has been reported as a major cause of food poisoning in addition to pathogenic Escherichia coli, norovirus, and Salmonella [8]; however, there have been no published reports regarding the use of whole- 
genome sequencing (WGS) to investigate $S$. aureus outbreaks. In this study, we describe a practical and effective approach to determine the source and routes of infection in a foodborne S. aureus outbreak that occurred at a village festival in Korea in November 2018 by combining epidemiological studies and WGS.

\section{Materials \& Methods \\ Epidemiological study}

On November 30, 2018, the local public health center was notified of a suspected foodborne outbreak at 6:30 pm. On that day, a village festival with 200 participants occurred on Wonsan Island, which is located in Boryeong, Chungcheongnam-do, Korea. Food was served at 11:00 am by a caterer providing a buffet of panfried fish filet, rice cakes, spicy pork and several other dishes. A case-control study was conducted to find evidence for or against potential sources. A case was defined as any person with an acute onset of symptoms after consuming the commercially catered buffet for lunch on the day of the festival. A control was defined as anyone who attended the buffet on that day but had not presented any symptoms. The food consumption, sociodemographic and clinical data of patients were collected by a structured questionnaire [22]. Epidemic curves were created using data from 26 symptomatic subjects. The odds ratio $(O R)$ and $95 \%$ confidence interval (CI) were calculated to assess the association between each food and the case or control group, in which values for unknown or no responses were excluded from analysis. The data were analyzed using Microsoft Excel 2013 (Microsoft Corp., USA).

\section{Laboratory tests}

Rectal swab samples collected from 26 members of the case group and 30 food handlers as well as 10 samples from the leftover food, 4 environmental surface samples, and 5 water specimens were transported to the provincial public health laboratory. Briefly, the pretreated samples were inoculated in selective and enriched media to identify the presence of bacteria (Salmonella spp., Shigella spp., S. aureus, Bacillus cereus, Vibrio spp., Clostridium perfringens, Listeria monocytogenes, pathogenic E. coli, Campylobacter jejuni/coli, and Yersinia enterocolitica) and viruses (Norovirus, Astrovirus, Enteric Adenovirus, Rotavirus, and Sapovirus). Additionally, preserved rice cakes $(n=3)$, which were prepared in a shop located approximately 100 km away from the location of the outbreak, were tested for bacteria and viruses (Supplementary Table 1). Suspected colonies were isolated on a mannitol salt agar plate and identified as $S$. aureus by the Vitek 2 system used with 
the gram-positive (GP) identification card (bioMérieux, France) and API Staph identification kit (bioMérieux, France) [22]. For the correlation analysis between the strains isolated from humans and foods, all isolated $S$. aureus were subjected to pulsed-field gel electrophoresis (PFGE) [23], and the results were plotted in a dendrogram with the dice similarity index method using BioNumerics v.5.1 (Applied Maths, Belgium). Antimicrobial susceptibility testing (AST) was also conducted by using the broth microdilution method with the Sensititre panel and interpreted according to the CLSI guidelines [24]. Susceptibility to 18 antimicrobial agents was tested: erythromycin (ERY), clindamycin (CLI), quinupristin/dalfopristin (SYN), daptomycin (DAP), vancomycin (VAN), tetracycline (TET), ampicillin (AMP), gentamicin (GEN), levofloxacin (LEVO), linezolid (LZD), ceftriaxone (AXO), streptomycin (STR), penicillin (PEN), rifampin (RIF), gatifloxacin (GAT), ciprofloxacin (CIP), trimethoprim/sulfamethoxazole (SXT), and oxacillin (OXA). The strains for PFGE and AST were cultured in trypticase soy agar for 20 hours at $37^{\circ} \mathrm{C}$.

\section{Whole-genome sequencing}

We analyzed $S$. aureus isolates by using WGS. For WGS, genomic DNA was extracted using a DNeasy Blood \& Tissue kit (Qiagen, USA) according to the manufacturer's instructions, and sample libraries were prepared using a Nextera DNA Flex library preparation kit (Illumina, Inc., USA). WGS was performed on an Illumina MiSeq platform using v3 reagent kits generating $2 \times 300$ bp paired-end reads (Illumina, USA) to obtain an average genome coverage greater than 100x for all the solates. Sequence reads were quality filtered and trimmed using BBDuk (http://jgi.doe.gov/data-and-tools/bb-tools/) and de novo assembled into contigs using SPAdes v3.12.0 [25]. The assembled WGS contigs were submitted to multilocus sequence typing (MLST) 2.0 on the Center for Genomic Epidemiology (CGE) website [26] to identify the multilocus sequence types (STs). The isolates for which the ST was classified as unknown were assigned using the S. aureus MLST database (https://pubmlst.org/). SCCmec typing was performed using WGS and SCCmec finder 1.2 on the CGE website in addition to multiplex PCR methods [27]. The presence of $S$. aureus virulence genes was identified in the VFDB database [28] using the BLAST tool of Geneious Prime 2019.2.1 [29]. The genes encoding enterotoxins were screened according to a previous report, which included a total of 99 sequences of staphylococcal enterotoxins and their variants [30].

\section{Phylogenetic analysis}


Sequences were mapped to S. aureus NCTC 8325, accession number NC_007795.1, as a reference genome using BioNumerics version 7.6 (Applied Maths, Belgium). A set of SNPs was deduced for each genome sequence data set using the BioNumerics whole genome SNP (wgSNP) module. The refined SNP matrix was used to generate a minimum spanning tree using the maximum bootstrap maximum likelihood approach, yielding the phylogenetic tree.

\section{Results}

\section{Epidemiological investigation}

The case-control study involved 86 participants, including 26 participants with symptoms and 60 participants without symptoms, and all 86 participants completed the questionnaires. The outbreak affected 26 out of 200 people who attended the festival (attack rate of 13\%). Approximately one hour after ingesting a variety of foods, the first guests complained about abdominal pain and diarrhea, and there were no additional patients by approximately 6:00 pm. The mean incubation time was 4.7 hours (at least 1 hour, at most 6.2 hours) (Figure 1). None of the patients had serious symptoms. The symptoms included vomiting (88.5\%), diarrhea (69.2\%), nausea $(42.3 \%)$, abdominal pain (42.3\%), and chills (34.6\%). The results from the case-control study implicated that the odds of having been exposed to rice cakes in those who had symptoms was 8.87 times that of people who did not consume rice cakes (OR (95\% CI): 8.87 (1.96-40.11)), suggesting that rice cakes were the most likely vehicle of food poisoning served for lunch on the festival day (Table 1).

\section{Microbiological analysis}

Among the 56 rectal swabs collected from the patients and food handlers, 17 samples were positive for $S$. aureus. Among the 17 S. aureus isolates, one was isolated from a food handler who was involved in the packing of the rice cakes. In addition, $7 \mathrm{~S}$. aureus isolates were isolated from leftover food; in particular, three were isolated from the rice cake shop. No microorganisms were found in the environmental samples, including those from cookware and drinking water (Supplementary Table 1). The antimicrobial susceptibility test of all $24 S$. aureus isolates indicated that they were all susceptible to all antimicrobials tested.

\section{Sequencing analysis}


Based on whole-genome analysis, the STs could be assigned for 21 (87.5\%) of the 24 isolates, and a S. aureus protein A (spa) type was assigned for all 24 isolates. There were two spa types, t304 and t11652, among the 24 isolates, and spa $\mathrm{t} 304$ was the predominant type $(\mathrm{n}=21)$. All spa type $\mathrm{t} 304$ isolates belonged to ST6, while the ST of spa t11652 was unknown. The unknown ST was assigned as ST5870. Furthermore, we performed a SCCmec analysis for all 24 isolates, and none of the isolates tested positive for the mecA gene [31], which was compatible with the PCR results. The screening of various virulence genes based on the WGS data indicated that virulence genes, including $\operatorname{sel} X, h l b, h l d, h l g A, h l g B, h l g \mathrm{C}, h l y / h l a, l u k D, l u k E$, aur, geh, sspA, sspB, and $s s p C$, were positive in the isolates belonging to ST6. On the other hand, the other three isolates were positive for sea, seg, sei, sem, sen, seo, seu, sak, sn, hld, hlgA, hlgC, geh, sspB, and sspC. Notably, the staphylococcal enterotoxin-like W (selW) gene was identified in all 24 isolates (Figure 2, Supplementary Table 2).

\section{Phylogenetic tree based on SNPs}

The genetic relatedness of the 24 isolates is shown in a phylogenetic tree (Figure 2). The whole-genome phylogeny of all the isolates showed that they clustered into two distinct groups, and their grouping was completely dependent on their spa type and ST. The first group contained the isolates that belonged to ST6 and spa type t304. In this group, genotypically identical strains were isolated from 13 patients, the food handler and 7 leftover foods (rice cakes, spicy pork and pan-fried fish filet). The second group belonged to spa type t11652 and ST5870. Based on the topology of the tree and the lengths of the branches, we confirmed that the two groups were not genetically related. The SNP-based cluster analysis of the isolates belonging to ST6 and spa type $\mathrm{t} 304$ revealed that the maximum SNP distances were no greater than 15 SNPs (Figure 3), suggesting that these isolates were within the range of epidemiological linkage.

\section{Discussion}

Outbreak investigations typically rely on spatiotemporal data and PFGE, but WGS is also being increasingly used $[32,33]$. WGS can provide higher discriminatory power than any other typing method. Therefore, applying WGS in outbreak investigations can enable the detection of origins of contamination quickly and accurately, resulting in a substantial reduction in the size of an outbreak [20,34]. 
The case-control study revealed that the outbreak was linked to the consumption of rice cakes. The identification of $S$. aureus in a rice cake product from the rice cake shop and on an employee who worked in the rice cake shop strongly suggested that the employee was an $S$. aureus carrier. There was no evidence of contamination in the drinking water, and the risk of illness from eating the other food items served was minimal and not statistically significant.

In an attempt to understand the routes of transmission, we obtained genomic data from the $S$. aureus isolates. In silico typing and the phylogenetic tree based on the SNPs of all of the isolates indicated that there were two distinct clonal strains involved in this outbreak. The three isolates that belonged to ST5870-t11652 were genetically distant from isolates that were isolated from the food handler and leftover food, suggesting that they were unrelated to the consumption of contaminated food and thus could be excluded from this outbreak case. Note that the spa type t11652 was previously only reported from the isolates in Ireland from 2012-2013 according to the Ridom SpaServer (https://spa.ridom.de/spa-t11652.shtml). The other 21 S. aureus ST6-t304 isolates formed the predominant lineage, and they were closely related based on the phylogenetic tree. These results suggested that the rice cakes were contaminated by the food handler and that the other food was crosscontaminated by the rice cakes. Therefore, the WGS analysis supports the findings of the previous epidemiological investigation. According to previous reports, the $S$. aureus ST6 strains were the most common strains associated with foodborne outbreaks in China $[35,36]$. In particular, ST6-t304 S. aureus was the most frequent clone and was related to four outbreaks in Hangzhou, China [37]. On the other hand, in Korea, the MLST of S. aureus related to food poisoning has varied, including ST6, ST1, ST59 and ST30, which were the major clones [38]. ST6-t304 S. aureus strains were isolated from the general German population as well [39]. Additionally, ST6-t304 methicillin-resistant S. aureus was also isolated from hospitals in the United Arab Emirates [40] and from retail food in Singapore [41].

This study has some limitations. A retrospective cohort study that included all participants in the village festival was not performed. The outbreak occurred on an island where the only means of transportation was by ship, and many participants were elderly people. Therefore, it was difficult to interview all of the participants. In addition, a rapid investigation of the environmental surfaces on which food was prepared was not conducted, resulting in no detection of $S$. aureus from environmental samples. 
Here, we showed that WGS could be applied for SFP outbreak investigation and detection. These data are in agreement with other recent reports $[32,42]$ that WGS can be a tool to support outbreaks and epidemiological investigations.

\section{Sequence data access}

The genomic data of this study have been deposited with links to BioProject accession number PRJNA632636 in the DDBJ BioProject database.

\section{Acknowledgements}

This work was supported by a grant from the Korea Centers for Disease Control and Prevention (grant number 4837-311-210).

\section{Conflicts of interest}

The authors have declared no conflicts of interest.

\section{References}

(1) Hoffmann S, et al. Attribution of global foodborne disease to specific foods: Findings from a World Health Organization structured expert elicitation. PloS one 2017; 12(9): e0183641.

(2) Ercoli L, et al. Investigation of a Staphylococcal Food Poisoning Outbreak from a Chantilly Cream Dessert, in Umbria (Italy). Foodborne pathogens and disease 2017; 14(7): 407-413.

(3) Hennekinne JA, De Buyser ML, Dragacci S. Staphylococcus aureus and its food poisoning toxins: characterization and outbreak investigation. FEMS microbiology reviews 2012; 36(4): 815-836.

(4) Mossong J, et al. Investigation of a staphylococcal food poisoning outbreak combining case-control, traditional typing and whole genome sequencing methods, Luxembourg, June 2014. Euro surveillance : bulletin Europeen sur les maladies transmissibles = European communicable disease bulletin 2015; 20(45).

(5) Scallan E, et al. Foodborne illness acquired in the United States--unspecified agents. Emerging infectious diseases 2011; 17(1): 16-22.

(6) ECDC) EFSAaECfDPaCEa. The European Union One Health 2018 Zoonoses Report. EFSA J 2019. 
Foodborne pathogens and disease 2016; 13(2): 101-107.

(8) Anon. Risk Assessment of Staphylococcus aureus in Dried fishes. National Institute of Food and Drug Safety Evaluation 2017.

(9) Le Loir Y, Baron F, Gautier M. Staphylococcus aureus and food poisoning. Genetics and molecular research: GMR 2003; 2(1): 63-76.

(10) Ono HK, et al. Identification and Characterization of a Novel Staphylococcal Emetic Toxin. Applied and environmental microbiology 2015; 81(20): 7034-7040.

(11) Fetsch A, et al. Staphylococcus aureus food-poisoning outbreak associated with the consumption of ice-cream. International journal of food microbiology 2014; 187: 1-6.

(12) Gallina S, et al. Staphylococcal poisoning foodborne outbreak: epidemiological investigation and strain genotyping. Journal of food protection 2013; 76(12): 2093-2098.

(13) Castro A, et al. Food handlers as potential sources of dissemination of virulent strains of Staphylococcus aureus in the community. Journal of infection and public health 2016; 9(2): 153-160.

(14) Tranter HS. Foodborne staphylococcal illness. Lancet 1990; 336(8722): 1044-1046.

(15) Li HT, et al. Factors associated with the outcome of life-threatening necrotizing pneumonia due to community-acquired Staphylococcus aureus in adult and adolescent patients. Respiration; international review of thoracic diseases $2011 ; \mathbf{8 1}(6)$ : 448-460.

(16) Vickery TW, Ramakrishnan VR, Suh JD. The Role of Staphylococcus aureus in Patients with Chronic Sinusitis and Nasal Polyposis. Current allergy and asthma reports 2019; 19(4): 21.

(17) Rohde H, et al. Open-source genomic analysis of Shiga-toxin-producing E. coli O104:H4. The New England journal of medicine 2011; 365(8): 718-724.

(18) Harris SR, et al. Whole-genome sequencing for analysis of an outbreak of meticillin-resistant Staphylococcus aureus: a descriptive study. The Lancet Infectious diseases 2013; 13(2): 130-136.

(19) Bartels MD, et al. Comparing whole-genome sequencing with Sanger sequencing for spa typing of methicillin-resistant Staphylococcus aureus. Journal of clinical microbiology 2014; 52(12): 4305-4308.

(20) van der Werf MJ, Kodmon C. Whole-Genome Sequencing as Tool for Investigating International Tuberculosis Outbreaks: A Systematic Review. Frontiers in public health 2019; 7: 87. 
Gordon NC, et al. Whole-Genome Sequencing Reveals the Contribution of Long-Term Carriers in Staphylococcus aureus Outbreak Investigation. Journal of clinical microbiology 2017; 55(7): 2188-2197.

(KCDC) KCfDCaP. 2018 Guideline for control of waterborne and food-borne disease Anon. Centers for Disease Control and Prevention. Unified pulsed field gel electrophoresis (PFGE) protocol for Gram-positive bacteria at www.cdc.gov/hai/pdfs/labSettings/Unified_PFGE_Protocol.pdf. In, 2012. (24) CLSI. Performance standards for antimicrobial susceptibility testing. 29th ed. Clinical and Laboratory Standards Institute; Wayne, PA: 2019. CLSI supplement M100.

Bankevich A, et al. SPAdes: a new genome assembly algorithm and its applications to single-cell sequencing. Journal of computational biology : a journal of computational molecular cell biology 2012; 19(5): 455-477.

Larsen MV, et al. Multilocus sequence typing of total-genome-sequenced bacteria. Journal of clinical microbiology 2012; 50(4): 1355-1361.

Stegger M, et al. Rapid detection, differentiation and typing of methicillin-resistant Staphylococcus aureus harbouring either mecA or the new mecA homologue mecA(LGA251). Clinical microbiology and infection : the official publication of the European Society of Clinical Microbiology and Infectious Diseases 2012; 18(4): 395-400.

(28) Chen L, et al. VFDB 2016: hierarchical and refined dataset for big data analysis--10 years on. Nucleic acids research 2016; 44(D1): D694-697.

(29) Kearse M, et al. Geneious Basic: an integrated and extendable desktop software platform for the organization and analysis of sequence data. Bioinformatics 2012; 28(12): 1647-1649.

(30) Macori G, et al. Genome-Wide Profiling of Enterotoxigenic Staphylococcus aureus Strains Used for the Production of Naturally Contaminated Cheeses. Genes 2019; 11(1).

International Working Group on the Classification of Staphylococcal Cassette Chromosome E. Classification of staphylococcal cassette chromosome mec (SCCmec): guidelines for reporting novel SCCmec elements. Antimicrobial agents and chemotherapy 2009; 53(12): 4961-4967.

(32) Durand G, et al. Routine Whole-Genome Sequencing for Outbreak Investigations of Staphylococcus aureus in a National Reference Center. Frontiers in microbiology 2018; 9: 511.

(33) Schjorring S, et al. Cross-border outbreak of listeriosis caused by cold-smoked salmon, revealed by integrated surveillance and whole genome sequencing (WGS), Denmark and France, 2015 to 2017. Euro 
surveillance : bulletin Europeen sur les maladies transmissibles = European communicable disease bulletin $2017 ; 22(50)$.

(34) van Beek J, et al. Tracing local and regional clusters of carbapenemase-producing Klebsiella pneumoniae ST512 with whole genome sequencing, Finland, 2013 to 2018. Euro surveillance : bulletin Europeen sur les maladies transmissibles = European communicable disease bulletin 2019; 24(38).

(35) Li G, et al. Staphylococcus aureus ST6-t701 isolates from food-poisoning outbreaks (2006-2013) in Xi'an, China. Foodborne pathogens and disease 2015; 12(3): 203-206.

(36) Yan X, et al. Characterization of Staphylococcus aureus strains associated with food poisoning in Shenzhen, China. Applied and environmental microbiology 2012; 78(18): 6637-6642.

Chen Q, Xie S. Genotypes, Enterotoxin Gene Profiles, and Antimicrobial Resistance of Staphylococcus aureus Associated with Foodborne Outbreaks in Hangzhou, China. Toxins 2019; 11(6).

(38) Cha JO, et al. Molecular analysis of Staphylococcus aureus isolates associated with staphylococcal food poisoning in South Korea. Journal of applied microbiology 2006; 101(4): 864-871.

(39) Becker K, et al. Staphylococcus aureus from the German general population is highly diverse. International journal of medical microbiology : IJMM 2017; 307(1): 21-27.

(40) Sonnevend A, et al. Change in meticillin-resistant Staphylococcus aureus clones at a tertiary care hospital in the United Arab Emirates over a 5-year period. Journal of clinical pathology 2012; 65(2): 178-182. (41) Aung KT, et al. Prevalence of methicillin-resistant Staphylococcus aureus (MRSA) in retail food in Singapore. Antimicrobial resistance and infection control 2017; 6: 94.

(42) Manara S, et al. Whole-genome epidemiology, characterisation, and phylogenetic reconstruction of Staphylococcus aureus strains in a paediatric hospital. Genome medicine 2018; 10(1): 82.

(43) Letunic I, Bork P. Interactive Tree Of Life v2: online annotation and display of phylogenetic trees made easy. Nucleic acids research 2011; 39(Web Server issue): W475-478. 
Figure legends

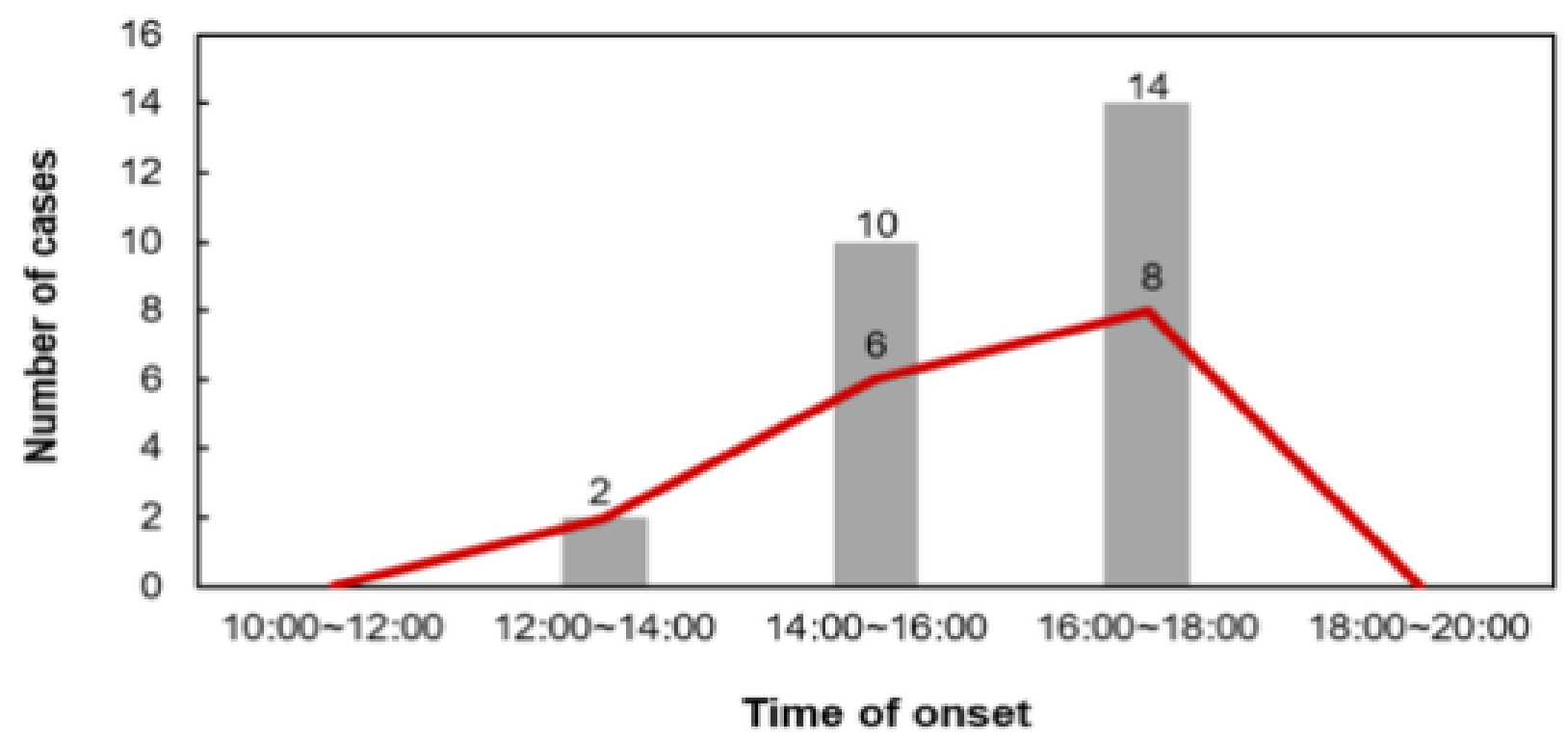

Suspected cases Laboratory-confirmed cases

Figure 1. Cases of food poisoning gastroenteritis by onset of illness during the outbreak on November 30, 2018. 


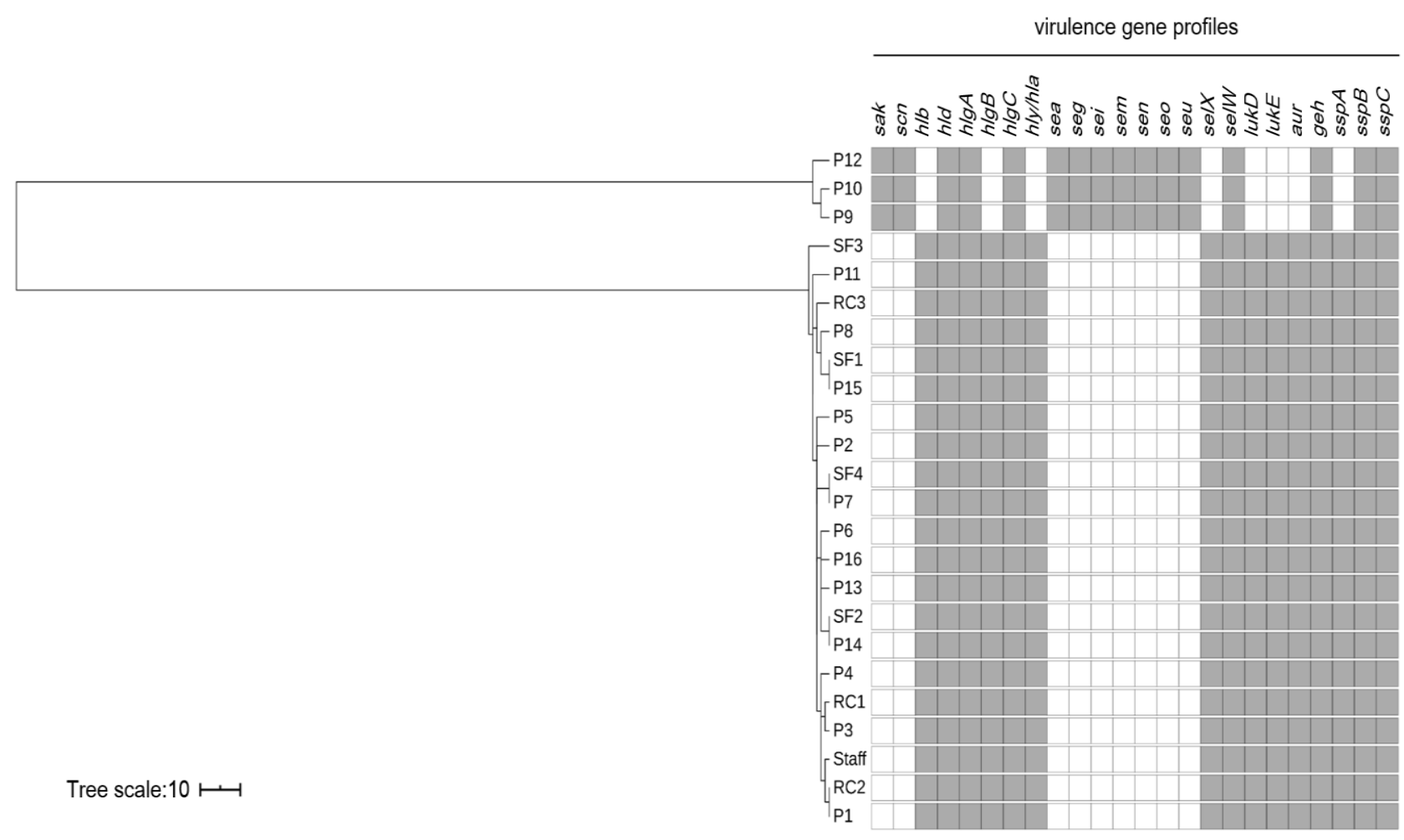

Figure 2. The phylogenetic tree based on SNP analysis using the BioNumerics pipeline. The data sets on virulence genes were integrated using iTOL [43]. Eight enterotoxin genes (sea, seg, sei, sem, sen, seo, seu, and selW) were detected in the isolates belonging to ST5870-t11652, and two enterotoxin genes (selX and selW) were detected in the isolates belonging to ST6-t304. The selW gene was identified in all 24 isolates. 


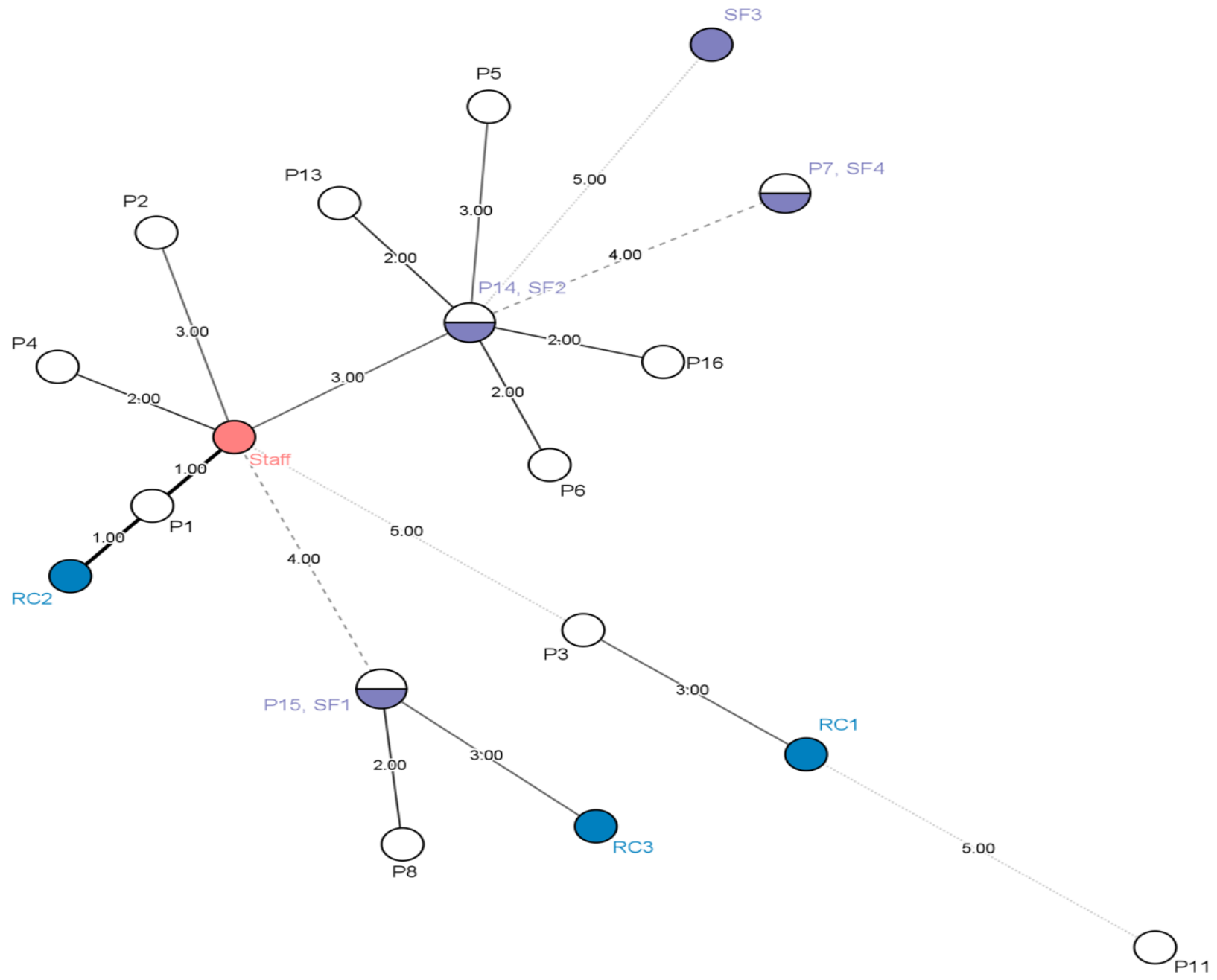

Figure 3. The MSTs based on SNPs of ST6-t304 S. aureus isolates. The isolates that were isolated from patients (P), served foods (SFs), and rice cakes (RCs) in the cake shop are colored white, violet, and blue, respectively. The isolate from the food handler is highlighted in pink. Detailed information about the rice cakes and served foods is as follows: $\mathrm{RC} 1$, rice cake 1 in the cake shop; $\mathrm{RC} 2$, rice cake 2 in the cake shop; $\mathrm{RC} 3$, rice cake 3 in the cake shop; SF1, served rice cake; SF 2, served rice cake with mugwort; SF3, served pan-fried fish filet; and SF4, served spicy pork. 
Table 1. Results from analytical case-control study of food exposure in the staphylococcal food-poisoning outbreak in Korea, November 2018

\begin{tabular}{|c|c|c|c|c|c|c|c|c|c|c|}
\hline \multirow{2}{*}{ Foods } & \multicolumn{3}{|c|}{ Patients } & \multicolumn{3}{|c|}{ Controls } & \multirow{2}{*}{ p-value } & \multirow{2}{*}{ Odds ratio } & \multicolumn{2}{|c|}{ IC $95 \%^{\mathrm{a}}$} \\
\hline & Exposed & Unexposed & Total & Exposed & Unexposed & Total & & & Lower & Upper \\
\hline Spicy pork & 9 & 17 & 26 & 14 & 65 & 79 & 0.0708 & 2.458 & 0.9105 & 6.6358 \\
\hline Fried pork & 12 & 14 & 26 & 30 & 49 & 79 & 0.4603 & 1.4 & 0.5721 & 3.4262 \\
\hline Seasoned skate & 18 & 8 & 26 & 47 & 32 & 79 & 0.3702 & 1.5319 & 0.5947 & 3.9463 \\
\hline Cold jellyfish salad & 14 & 12 & 26 & 27 & 53 & 80 & 0.0676 & 2.2901 & 0.9315 & 5.6306 \\
\hline Panfried fish filet & 12 & 14 & 26 & 20 & 59 & 79 & 0.0453 & 2.5286 & 1.0048 & 6.3633 \\
\hline Japchae $^{b}$ & 16 & 10 & 26 & 38 & 41 & 79 & 0.2344 & 1.7263 & 0.6984 & 4.2674 \\
\hline Steamed rice & 20 & 6 & 26 & 65 & 15 & 80 & 0.6306 & 0.7692 & 0.2635 & 2.2454 \\
\hline Kimchi soup & 14 & 12 & 26 & 39 & 41 & 80 & 0.6516 & 1.2265 & 0.5052 & 2.9777 \\
\hline Stir-fried anchovies & 7 & 19 & 26 & 13 & 66 & 79 & 0.2384 & 1.8704 & 0.6539 & 5.3507 \\
\hline Seasoned vegetables & 12 & 14 & 26 & 31 & 48 & 79 & 0.5341 & 1.3272 & 0.5431 & 3.2435 \\
\hline Clam soup & 17 & 9 & 26 & 53 & 26 & 79 & 0.873 & 0.9266 & 0.364 & 2.3588 \\
\hline Rice cake & 24 & 2 & 26 & 46 & 34 & 80 & 0.0011 & 8.8696 & 1.9611 & 40.1138 \\
\hline Pork slice & 18 & 8 & 26 & 48 & 31 & 79 & 0.4381 & 1.4531 & 0.5634 & 3.7476 \\
\hline Oyster & 8 & 18 & 26 & 15 & 64 & 79 & 0.2077 & 1.8963 & 0.6942 & 5.1798 \\
\hline Kimchi & 7 & 19 & 26 & 27 & 52 & 79 & 0.4929 & 0.7096 & 0.2654 & 1.8971 \\
\hline
\end{tabular}




\begin{tabular}{|c|c|c|c|c|c|c|c|c|c|c|} 
Drinking water & 11 & 15 & 26 & 28 & 51 & 79 & 0.5298 & 1.3357 & 0.5407 & 3.2997 \\
\hline
\end{tabular}

${ }^{\mathrm{b}}$ Japchae: mixed dish of boiled bean threads, stir-fried vegetables, and shredded meat. 